The submitted manuscript has been authored by a contractor of the U.S. Government under contract No. DE-AC05-960R22464. Accordingly, the U.S. Govemment retains a nonexclusive, royalty-free license to publish or reproduce the published form of this contribution, or allow others to do so, for U.S. Government purposes.

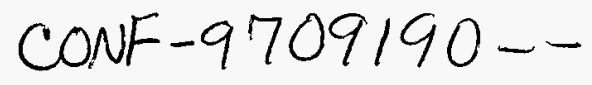

1

\title{
ORLANDO - Oak Ridge Large Neutrino Detector
} W. Bugga , H. Cohn
and R. Svoboda ${ }^{a}$

aUniversity of Tennessee, Knoxville, TN 37996

OSTI

baak Ridge National Laboratory, Oak Ridge, TN 37831

'Southern University, Baton Rouge, LA 70813

${ }^{\mathrm{d}}$ Louisiana State University, Baton Rouge, LA 70803

We discuss a proposal for construction of an Oak Ridge LArge Neutrino DetectOr (ORLANDO) to search for neutrino oscillations at the Spallation Neutron Source (SNS). A $4 \mathrm{MW}$ SNS is proposed to be built at the Oak Ridge National Laboratory with the first stage to be operative around 2006. It will have two target stations, which makes it possible with a single detector to perform a neutrino oscillation search at two different distances. Initial plans for the placement of the detector and the discovery potential of such a detector are discussed.

\section{INTRODUCTION}

At spallation sources neutrinos (with energies $>10 \mathrm{MeV}$ ) are produced predominantly by stopping and subsequent decay of pions and muons originating in the spallation target. Spallation sources driven by protons with energy around $1 \mathrm{GeV}$ have several advantages over other types of neutrino sources: (1) a well-understood neutrino flavor spectrum; (2) no contamination from kaon decays; (3) no contamination from $\nu-N$ pion production in the detector; and (4) a predictable energy spectrum. Previously, spallation-sourcebased neutrino oscillation searches have typically been limited by low intensity (e.g. KARMEN at ISIS [1-5]), long beam extraction times (e.g. LSND at LAMPF [6]), single-distance running (e.g. KARMEN and LSND), and limited statistics (e.g. KARMEN and LSND). Recently the LSND collaboration published their result of a search for $\bar{\nu}_{\mu} \rightarrow \bar{\nu}_{e}$ transitions at LAMPF[6]. They have detected $22 \bar{\nu}_{e}$ events with an expected background of 4.6 events, which might be interpreted as evidence for neutrino oscillations. This extraordinary result should be verified, and if confirmed, mixing parameters should be measured with the highest possible accuracy.

-E-mail:efremenk@@utkux1.utk.edu
In this paper we discuss the advantages of the proposed Oak Ridge Spallation Neutron Source (SNS) [7] for solving this problem.

\section{SPALLATION NEUTRON SOURCE}

The proposed Oak Ridge SNS [7] would consist of a $1 \mathrm{GeV}$ proton linear accelerator, two proton storage rings, two target stations $(60 \mathrm{~Hz}$ and $10 \mathrm{~Hz}$ ) with short beam extraction time $(0.5 \mu s)$ which will ultimately deliver beam currents of 4 $\mathrm{mA}$ and $1 \mathrm{~mA}$, respectively, and several beam stops with lower currents for testing and tuning. This facility is similar to the European Spallation Source (ESS) facility [8], however, the ESS is planned to come into operation no earlier than several years after SNS. These machines will produce as a by-product a powerful flux of neutrinos and because of the heavy target material are ideally suited for a neutrino flavor oscillation search.

Fluxes (for neutrino energies in the range 10 $60 \mathrm{MeV}$ ) expected at the NSNS for 1MW (4MW) beams will be roughly 1.3 (5) times those obtained at LAMPF [9]. These flux increases result from enhanced pion production at $1000 \mathrm{MeV}$ versus $800 \mathrm{MeV}$ and from the higher beam power. Two independent target stations operating simultaneously, but within different time slots, will 


\section{DISCLAIMER}

This report was prepared as an account of work sponsored by an agency of the United States Government. Neither the United States Government nor any agency thereof, nor any of their employees, makes any warranty, express or implied, or assumes any legal liability or responsibility for the accuracy, completeness, or usefulness of any information, apparatus, product, or process disclosed, or represents that its use would not infringe privately owned rights. Reference herein to any specific commercial product, process, or service by trade name, trademark, manufacturer, or otherwise does not necessarily constitute or imply its endorsement, recommendation, or favoring by the United States Government or any agency thereof. The views and opinions of authors expressed herein do not necessarily state or reflect those of the United States Government or any agency thereof. 
allow simultaneous neutrino oscillation searches with the same detector at two different sourceto-detector distances. The short beam-time structure will make a non-beam-associated background contribution essentially negligible compared to other background sources. Heavy material (liquid mercury) proposed for use in the spallation targets has the advantage of being extremly effective for stopping and capture of $\pi^{-}$ and $\mu^{-}$, thus potentially reducing the rate of $\bar{\nu}_{e}$ production, and giving different time structures for $\bar{\nu}_{e}$ and $\bar{\nu}_{\mu}$. Rate of $\bar{\nu}_{e}$ production determines the principal background for the $\bar{\nu}_{\mu} \rightarrow \bar{\nu}_{e}$ transition search. Figure 1 shows the simulated energy and time distributions for $\bar{\nu}_{\mu}$ and $\bar{\nu}_{e}$.
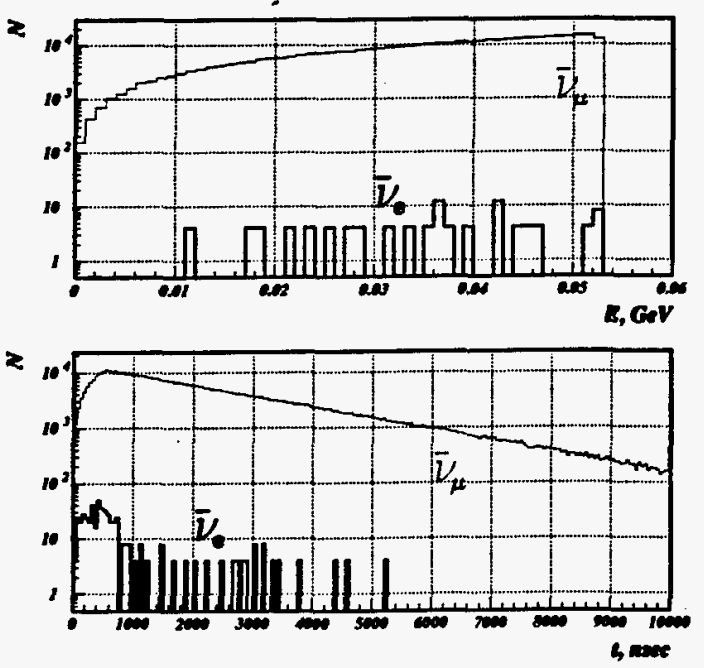

Figure 1. Energy and time spectra of $\bar{\nu}_{\mu}$ and $\bar{\nu}_{e}$ at a proposed Spallation Neutron Source.

The auxiliary beam stops of the SNS (with power $200 \mathrm{~kW}$ ), although operating at only a fraction of the total beam intensity or time, will each be as powerful as ISIS. They will provide additional neutrino sources which will be seen by the neutrino detector in different time slots. Con- struction of these beam stops can be optimized to enhance the production of energetic neutrinos from decay-in-flight pions and muons, while the main spallation targets will predominantly produce neutrinos from decay-at-rest.

It is anticipated that the first spallation target station will become available at $1 \mathrm{MW}$ in 2006 , with a first upgrade to $2 \mathrm{MW}$ in 2007 and a further upgrade to $4 \mathrm{MW}$ around 2010 . Second target station might be available around 2007.

\section{NEW NEUTRINO DETECTOR}

Plans for a new large neutrino detector at the SNS are being pursued by the ORLANDO (Oak Ridge LArge Neutrino DetectOr) Collaboration $[10,11]$. A new large liquid-scintillator-based detector with sensitive mass in the range of 1-2 kton is being discussed. We envisage the most appropriate position for detector in an underground hall, constructed as close to the first target station as possible. The bunker for neutrino detector would have to be completed before begining of the first target station construction. Initial engineering study indicates that the closest distance between the center of detector and spallation target should be approximately $48 \mathrm{~m}$ and corresponding distance to the second target $\sim 103$ meters. The distances from the detector to the auxiliary beam stops will be in the range of $110-145 \mathrm{~m}$.

Figure 2 shows the discovery potential of the proposed SNS-based neutrino detector in oscillation-parameter space $\Delta m^{2}$ vs $\sin ^{2} 2 \theta$. The discovery potential ( $3 \sigma$ signal contours) was derived from GEANT simulations of neutrino production in the target using the geometry and shielding layout of the spallation target station in its conceptual design[7]. The two contours correspond to the distances of a 1.5 -kton detector to two spallation target stations located at 48 and $103 \mathrm{~m}$ respectively. The power at each spallation target station was assumed in the calculation to be $1 \mathrm{MW}$ with a detector running time of 3 calendar years ( $70 \%$ beam time). Figure 2 also shows the oscillation-parameter region allowed by LSND results [6] and 90\% CL exclusion regions from Bugey and BNL E776 experiments [12,13].

The ORLANDO detector might also be de- 


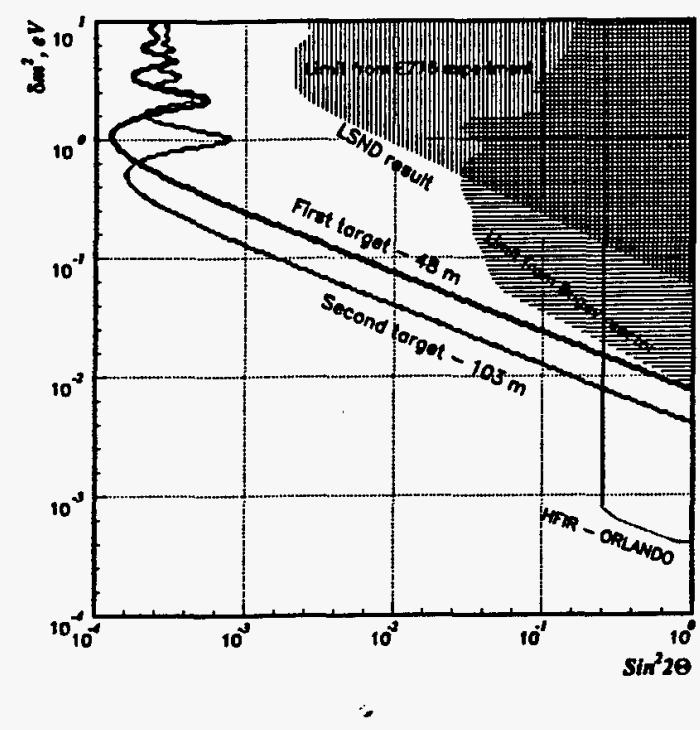

Figure 2. Sensitivity to $\bar{\nu}_{\mu} \rightarrow \bar{\nu}_{e}$ of a proposed SNS-based neutrino detector.

signed to be sensitive to the reactor neutrinos. In this scenario neutrino disappearance experiments, utilizing neutrinos from the HFIR (High Flux Isotope Reactor), located 4 miles to the south of the proposed site for SNS, could be performed. The discovery potential for this experiment, assuming a $2 \mathrm{MeV}$ detector threshold and 3 years of data taking, is also shown in Figure 2.

In conclusion, we would like to note that the proposed new SNS facility will offer an opportunity to advance the research pioneered at Los Alamos and Rutherford laboratories, by essentially combining the background-reducing shortbeam-pulse structure of ISIS with a beam power that is significantly higher than that at LAMPF. Neutrino beams at the SNS facility might be available several years before they become available at the ESS in Europe. Neutrino oscillation $\bar{\nu}_{\mu} \rightarrow \bar{\nu}_{e}$ sensitivities significantly higher than existing limits can be expected for a SNS-based detector of $\sim 1.5$ kton. Measurements at two distances should provide an essential check of the results. If the LSND result is reproducible, then we expect thousands of events in the region favored by the LSND collaboration. This would allow a precise measurements of mixing parameters.

This research is supported in part by the Oak Ridge National Laboratory managed by Lockheed Martin Energy Research Corp. for the U.S. Department of Energy under contract number DEAC05-96OR22464.

\section{REFERENCES}

1. G.Drexlin et al., Nucl. Instrum. Meth. A 289 (1990) 490.

2. B.Bodmann et al., Phys. Lett. B 267 (1991) 321.

3. B.Bodmann et al., Phys. Lett. B 280 (1992) 198.

4. B.Zeitnitz et al., Prog. Part. Nucl. Phys. 32 (1994) 351.

5. B.Armbruster et al., Phys. Lett. B 348 (1995) 19.

6. C.Athanassopoulos et al., Phys. Rev. Lett. 77 (1996) 3082.

7. The NSNS Collaboration, May 97, National Spallation Neutron Source Conceptual Design Report Vol. 1\&2 (ORNL, Oak Ridge: Tennessee).

8. March 1997, ESS A Next Generation Neutron Source for Europe The ESS Technical Study Report Volumes 1-3.

9. C.Athanassopoulos et al., Phys. Rev. C 54 (1996) 2685.

10. W.Bugg et al., Prospects for a neutrino oscillation experiment at the National Spallation Neutron Source submitted to the proceedings of the 6th Conference on the Intersections of Particle and Nuclear Physics (Big Sky: Montana) (1997).

11. W.Bugg et al., Prospects for a new neutrino oscillation search experiment at the NSNS submitted to the proceedings of the international workshop "Beyond the Desert" - Accelerator and Non-Accelerator Approaches, Castle Ringberg, Tegernsee, Germany, June 8-14, 1997.

12. B.Achkar et al., Nucl. Phys. B 434 (1995) 503.

13. L.Borodovsky et al., Phys. Rev. Lett. 68 (1992) 274. 


\section{M98003177}

||||||||||||||||||||||||||||||||||||||||||||||||||||||||||

Report Number (14) ORNL/CP $-96 / 53$

CONF-9709190-

गubl. Date (11)

Sponsor Code (18)

199712

JC Category (19)

DOE/ER, XF

$U C-400, D O E / E R$ 